\title{
Preliminary Study of Space-Time Finite-Element Eddy-Current Analysis
}

\section{$\operatorname{AUTHOR}(S)$ :}

Niimi, Junichi; Mifune, Takeshi; Matsuo, Tetsuji

\section{CITATION:}

Niimi, Junichi ... [et al]. Preliminary Study of Space-Time Finite-Element Eddy-Current Analysis. IEEE Transactions on Magnetics 2016, 52(3): 7401804.

\section{ISSUE DATE:}

2016-03

URL:

http://hdl.handle.net/2433/217061

\section{RIGHT:}

(C) 2015 IEEE. Personal use of this material is permitted. Permission from IEEE must be obtained for all other uses, in any current or future media, including reprinting/republishing this material for advertising or promotional purposes, creating new collective works, for resale or redistribution to servers or lists, or reuse of any copyrighted component of this work in other works. 


\title{
Preliminary Study of Space-time Finite element Eddy-current Analysis
}

\author{
Junichi Niimi, Takeshi Mifune, Member, IEEE, and Tetsuji Matsuo, Member, IEEE \\ Graduate School of Engineering, Kyoto University, Kyoto 615-8510, Japan, niimi,junichi.57e@st.kyoto-u.ac.jp
}

\begin{abstract}
A space-time finite element method is developed for the eddy-current analysis that enables temporally-variant spatial finite elements arising in the analysis of moving conductors. We discuss the relationship between coordinate transformations and the space-time finite element in treating moving conductors. The space-time triangular-prism and parallelepiped finite elements are found to represent successfully the eddy current field with a moving conductor in space-time.
\end{abstract}

Index Terms_Eddy current, space-time finite element, motional electromotive force

\section{INTRODUCTION}

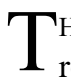
HE MAGNETIC field analysis including moving conductors requires the addition of the motional electromotive force (emf) or the combination of fixed and moving coordinate systems [1], [2]. This is based on the space-time nature of the Maxwell equations. However, time is usually formulated independently of space in the finite element electromagnetic field analysis. To obtain the time evolution for the electromagnetic field, a sequential scheme is generally used in a step-by-step manner with a uniform time step. With respect to the space-time nature of the Maxwell equations, however, it seems natural to generate the computational finite elements in space-time to handle temporally-variant spatial finite elements arising in the analysis of moving conductors.

Previous studies [3]-[5] have successfully introduced a temporal convolution for the symmetric formulation of spacetime finite element (FE) eddy-current analysis. However, the temporal convolution was only applied to the temporally uniform space-time elements and its application to the analysis of moving conductors is not straight forward.

We first construct the space-time finite elements and discuss the relation between the coordinate transformation and the motional emf in the finite element space-time. Second, a Galerkin-type space-time FE analysis is formulated to develop general polygon-type finite elements such as space-time prismatic and parallelepiped elements.

\section{SPACE-TIME FINITE ELEMENT EDDY-CURRENT ANALYSIS}

This section discusses the relationship between the spacetime finite element and the coordinate transformation in handling moving conductors.

\section{A. Coordinate transformation}

The governing equation for the eddy-current field including moving conductors is given as:

Manuscript received April 1, 2015; revised May 15, 2015 and June 1, 2015; accepted July 1, 2015. Date of publication July 10, 2015; date of current version July 31, 2015. Corresponding author: T. Matsuo (e-mail: matsuo.tetsuji.5u@kyoto-u.ac.jp).

Color versions of one or more of the figures in this paper are available online at http://ieeexplore.ieee.org.

Digital Object Identifier (inserted by IEEE).

$$
\begin{gathered}
\nabla \times(v \nabla \times \boldsymbol{A})=\boldsymbol{J}_{0}+\sigma\left(-\frac{\partial \boldsymbol{A}}{\partial t}-\nabla \varphi+\boldsymbol{V} \times \boldsymbol{B}\right), \\
\nabla \cdot\left[\sigma\left(-\frac{\partial \boldsymbol{A}}{\partial t}-\nabla \varphi+\boldsymbol{V} \times \boldsymbol{B}\right)\right]=0,
\end{gathered}
$$

where $\boldsymbol{A}$ is the vector potential, $\varphi$ the scalar potential, $\sigma$ the conductivity, $v$ the reluctivity, $\boldsymbol{B}=\operatorname{curl} \boldsymbol{A}$ the magnetic flux density, $\boldsymbol{J}_{0}$ the imposed current density, and $\boldsymbol{V}$ the velocity of the moving conductor. For simplicity, $\boldsymbol{V}$ is assumed constant and much smaller than the velocity of light. Using the Galilean transformation

$$
\left(\boldsymbol{r}^{\prime}, t^{\prime}\right)=(\boldsymbol{r}-\boldsymbol{V} t, t)
$$

(1) and (2) are written in the moving coordinate system [1] as

$$
\begin{gathered}
\nabla^{\prime} \times\left(\nu \nabla^{\prime} \times \boldsymbol{A}^{\prime}\right)=\boldsymbol{J}_{0}{ }^{\prime}+\sigma\left(-\frac{\partial \boldsymbol{A}^{\prime}}{\partial t}-\nabla^{\prime} \varphi^{\prime}\right), \\
\nabla^{\prime} \cdot\left[\sigma\left(-\frac{\partial \boldsymbol{A}^{\prime}}{\partial t^{\prime}}-\nabla^{\prime} \varphi^{\prime}\right)\right]=0
\end{gathered}
$$

where

$$
\begin{aligned}
& \boldsymbol{A}^{\prime}=\boldsymbol{A}, \quad \varphi^{\prime}=\varphi-\boldsymbol{V} \cdot \boldsymbol{A}, \quad \frac{\partial}{\partial t^{\prime}}=\frac{\partial}{\partial t}+\boldsymbol{V} \cdot \nabla, \\
& \boldsymbol{J}_{0}{ }^{\prime}=\boldsymbol{J}_{0}, \quad \nabla^{\prime}=\left(\frac{\partial}{\partial x^{\prime}}, \frac{\partial}{\partial y^{\prime}}, \frac{\partial}{\partial z^{\prime}}\right)=\left(\frac{\partial}{\partial x}, \frac{\partial}{\partial y}, \frac{\partial}{\partial z}\right)=\nabla
\end{aligned}
$$

The two-dimensional eddy-current analysis is discussed in this paper, where

$$
\boldsymbol{V}=\left(V_{x}, V_{y}, 0\right), \boldsymbol{A}=(0,0, A), \boldsymbol{J}_{0}=\left(0,0, J_{0}\right) .
$$

Consequently, from (6) and (7), we have

$$
\varphi^{\prime}=\varphi
$$

For simplicity, gauge condition $\varphi=0$ is assumed throughout. 


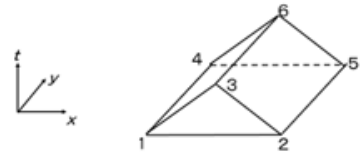

Fig. 1. Space-time triangular prismatic element

\section{B. Space-time finite elements}

Fig. 1 illustrates a prismatic element in $(x, y, t)$ space-time used in this study; here the node coordinates are given by

$$
\begin{aligned}
& x_{i+3}=x_{i}, \quad t_{i+3}=t_{i} \quad(i=1,2,3) \\
& y_{1}=y_{2}=y_{3}, \quad y_{4}=y_{5}=y_{6}
\end{aligned}
$$

The basis functions are given as

$$
\begin{gathered}
N_{i}=\frac{1}{2 \mathrm{vol}}\left(a_{i}+b_{i} x+c_{i} t\right)\left(y_{i+3}-y\right) \\
N_{i+3}=\frac{1}{2 \mathrm{vol}}\left(a_{i}+b_{i} x+c_{i} t\right)\left(y-y_{i}\right) \quad(i=1,2,3) \\
a_{1}=x_{2} t_{3}-x_{3} t_{2}, \quad b_{1}=t_{2}-t_{3}, \quad c_{1}=x_{3}-x_{2} \\
a_{2}=x_{3} t_{1}-x_{1} t_{3}, \quad b_{2}=t_{3}-t_{1}, \quad c_{2}=x_{1}-x_{3} \\
a_{3}=x_{1} t_{2}-x_{2} t_{1}, \quad b_{3}=t_{1}-t_{2}, \quad c_{3}=x_{2}-x_{1} \\
\Delta=\frac{1}{2}\left(a_{1}+a_{2}+a_{3}\right), \quad \mathrm{vol}=\Delta\left(y_{4}-y_{1}\right)
\end{gathered}
$$

For simplicity, the Galilean transformation with $\left(V_{x}, V_{y}\right)=(V$, $0)$ is assumed here. The basis functions $N_{i}{ }_{i}(i=1, \ldots, 6)$ in the transformed coordinates are similarly given by coefficients

$$
\begin{aligned}
& a_{i}{ }^{\prime}=a_{i}, \quad b_{i}{ }^{\prime}=b_{i}, \quad c_{i}{ }^{\prime}=c_{i}+V b_{i} \\
& \Delta^{\prime}=\Delta, \quad \text { vol }^{\prime}=\text { vol }
\end{aligned}
$$

Hence, we have

$$
\begin{aligned}
& \frac{\partial N_{i}{ }^{\prime}}{\partial t^{\prime}}=\frac{1}{2 \mathrm{vol}^{\prime}} c_{i}{ }^{\prime}\left(y_{i+3}{ }^{\prime}-y^{\prime}\right) \\
& =\frac{1}{2 \mathrm{vol}}\left(c_{i}+V b_{i}\right)\left(y_{i+3}-y\right)=\frac{\partial N_{i}}{\partial t}+V \frac{\partial N_{i}}{\partial x} \\
& \frac{\partial N_{i+3}{ }^{\prime}}{\partial t^{\prime}}=\frac{1}{2 \mathrm{vol}^{\prime}} c_{i}{ }^{\prime}\left(y^{\prime}-y_{i}{ }^{\prime}\right) \\
& =\frac{1}{2 \mathrm{vol}}\left(c_{i}+V b_{i}\right)\left(y-y_{i}\right)=\frac{\partial N_{i+3}}{\partial t}+V \frac{\partial N_{i+3}}{\partial x} \\
& (i=1,2,3)
\end{aligned}
$$

The two-dimensional eddy-current field is governed by

$$
\nabla(v \nabla A)=-J_{0}+\sigma\left(\frac{\partial A}{\partial t}+V \frac{\partial A}{\partial x}\right) .
$$

The vector potentials $A$ and $A$ ' are assumed to represent

$$
A=\sum_{i=1}^{6} A_{i} N_{i}, \quad A^{\prime}=\sum_{i=1}^{6} A_{i}{ }^{\prime} N_{i}{ }^{\prime} .
$$

From (13) and (15), it follows that

$$
\frac{\partial A}{\partial t}+V \frac{\partial A}{\partial x}=\frac{\partial A^{\prime}}{\partial t^{\prime}} .
$$

Consequently, (14) is equivalent to

$$
\nabla^{\prime}\left(v \nabla^{\prime} A\right)=-J_{0}{ }^{\prime}+\sigma \frac{\partial A^{\prime}}{\partial t^{\prime}} .
$$

even under the restriction to the space of functions (15).

The equivalence above under Galilean transformation holds generally for $V_{y} \neq 0$. This is because

$$
N(\boldsymbol{r}, t)=N\left(\boldsymbol{r}^{\prime}+\boldsymbol{V} t^{\prime}, t^{\prime}\right)=N^{\prime}\left(\boldsymbol{r}^{\prime}, t\right)
$$

is satisfied and consequently

$$
\left(\frac{\partial}{\partial t}+\boldsymbol{V} \cdot \nabla\right) N_{i}=\frac{\partial N^{\prime}}{\partial t^{\prime}}
$$

holds, where $N$ and $N^{\prime}$ are basis functions in the coordinate systems $(r, t)$ and $\left(\boldsymbol{r}^{\prime}, t^{\prime}\right)=(\boldsymbol{r}-\boldsymbol{V} t, t)$. If the space-time finite element satisfies (18), the addition of the motional electromotive force $\boldsymbol{V} \times \boldsymbol{B}$ to the electric field $-\partial \boldsymbol{A} / \partial t$ is equivalent to the Galilean transformation $\boldsymbol{r}^{\prime}=\boldsymbol{r}-\boldsymbol{V} t$.

\section{Formulation of space-time finite element method}

The Galerkin finite element method (FEM) is used to derive the weak form of (14). Multiplying (14) by the basis function $N_{i}$ as weighting function and integrating gives

$\iiint_{\Omega}\left[\frac{\partial}{\partial x}\left(v \frac{\partial A}{\partial x}\right) N_{i}+\frac{\partial}{\partial y}\left(v \frac{\partial A}{\partial y}\right) N_{i}\right] \mathrm{d} x \mathrm{~d} y \mathrm{~d} t$

$$
=\iiint_{\Omega} \sigma \frac{\partial A}{\partial t} N_{i}+\sigma V \frac{\partial A}{\partial x} N_{i} \mathrm{~d} x \mathrm{~d} y \mathrm{~d} t-\iiint_{\Omega} J_{0} N_{i} \mathrm{~d} x \mathrm{~d} y \mathrm{~d} t
$$

Integrating the left hand side by parts yields

$$
\begin{aligned}
\text { (L.H.S.) }=\int \oint v N_{i} & \left(\frac{\partial A}{\partial x} \mathrm{~d} y-\frac{\partial A}{\partial y} \mathrm{~d} x\right) \mathrm{d} t \\
& -\iiint_{\Omega}\left(v \frac{\partial A}{\partial x} \frac{\partial N_{i}}{\partial x}+v \frac{\partial A}{\partial y} \frac{\partial N_{i}}{\partial y}\right) \mathrm{d} x \mathrm{~d} y \mathrm{~d} t
\end{aligned}
$$

With the natural boundary condition, the weak form

$$
\begin{aligned}
\iiint_{\Omega}\left(v \frac{\partial A}{\partial x} \frac{\partial N_{i}}{\partial x}+v \frac{\partial A}{\partial y} \frac{\partial N_{i}}{\partial y}+\sigma \frac{\partial A}{\partial t} N_{i}\right. \\
\left.+\sigma V \frac{\partial A}{\partial x} N_{i}\right) \mathrm{d} x \mathrm{~d} y \mathrm{~d} t=\iiint_{\Omega} J_{0} N_{i} \mathrm{~d} x \mathrm{~d} y \mathrm{~d} t
\end{aligned}
$$

is obtained. Hence (22) produces the linear system of equations 


$$
([K]+[C]+[U])\{A\}=\{F\}
$$

where

$$
\begin{gathered}
K_{i j}=\iiint_{\Omega}\left(v \frac{\partial N_{i}}{\partial x} \frac{\partial N_{j}}{\partial x}+v \frac{\partial N_{i}}{\partial y} \frac{\partial N_{j}}{\partial y}\right) \mathrm{d} x \mathrm{~d} y \mathrm{~d} t \\
C_{i j}=\iiint_{\Omega}\left(\sigma N_{i} \frac{\partial N_{j}}{\partial t}\right) \mathrm{d} x \mathrm{~d} y \mathrm{~d} t \\
U_{i j}=\iiint_{\Omega}\left(\sigma V N_{i} \frac{\partial N_{j}}{\partial x}\right) \mathrm{d} x \mathrm{~d} y \mathrm{~d} t \\
F_{i}=\iiint_{\Omega}\left(J_{0} N_{i}\right) \mathrm{d} x \mathrm{~d} y \mathrm{~d} t
\end{gathered}
$$

\section{ANALYSIS RESULT OF EDDY-CURRENT FIELD}

\section{A. Square iron bar}

The magnetic flux of a square iron bar [Fig. 2(a)] is analyzed using the 3D space-time FEM, where the conductivity and relative permeability of the iron bar are $10^{6}$ $\mathrm{S} / \mathrm{m}$ and 300; the excitation frequency is $50 \mathrm{~Hz}$. The natural boundary condition is imposed at the symmetric boundaries, and $A=0$ at the outer boundaries.

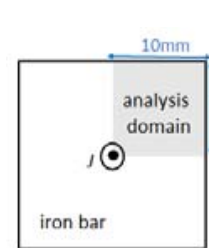

(a)

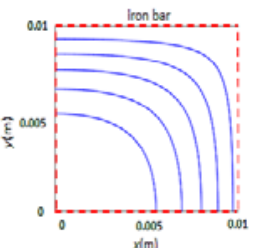

(b)
Fig. 2. Schematics of the square iron bar: (a) geometry and (b) magnetic flux lines obtained using the space-time FEM

The magnetic flux lines [Fig. 2(b)] were obtained using the space-time parallelepiped element. Fig. 3 shows the relative error compared with the analytical solution given by the Fourier expansion, and gives a comparison with the error given by the conventional FEM using the rectangular element. The space-time FEM is more accurate than the conventional FEM because for time integrations the latter uses the backward Euler scheme, which has lower-order precision than the scheme with space-time elements.

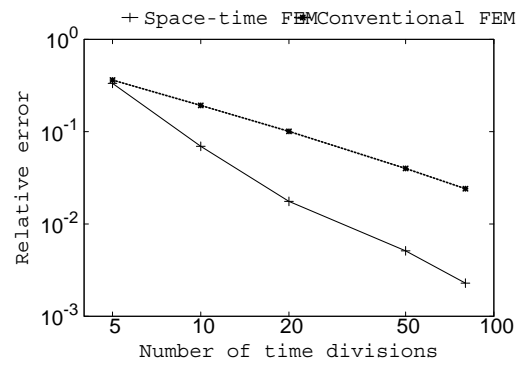

Fig. 3. Relative error given by the space-time FEM and the conventional FEM compared with the analytical solution

\section{B. Iron-cored inductor}

The iron-cored inductor (Fig. 4) is analyzed using the 3D space-time FEM, where the conductivity and relative permeability of the iron core are $10^{6} \mathrm{~S} / \mathrm{m}$ and 5000 , and the excitation frequency is $50 \mathrm{~Hz}$. The natural boundary condition is imposed at the symmetric boundaries, and $A=0$ at the outer boundaries.

Figs. 5(a) and (b) shows the magnetic flux lines obtained by the conventional FEM and by the space-time FEM using the triangular prism element. Fig. 5(c) illustrates the temporaryvariant spatial grid where the two spatial grids drawn by the

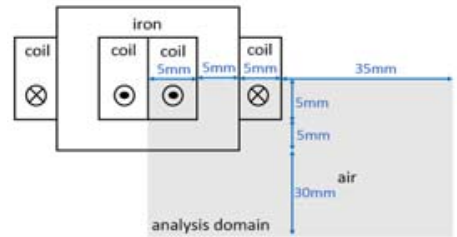

Fig. 4. Diagram of the iron-cored inductor

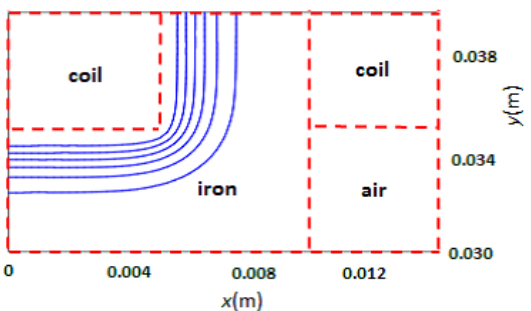

(a)

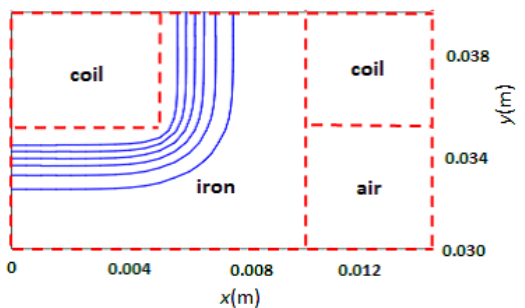

(b)

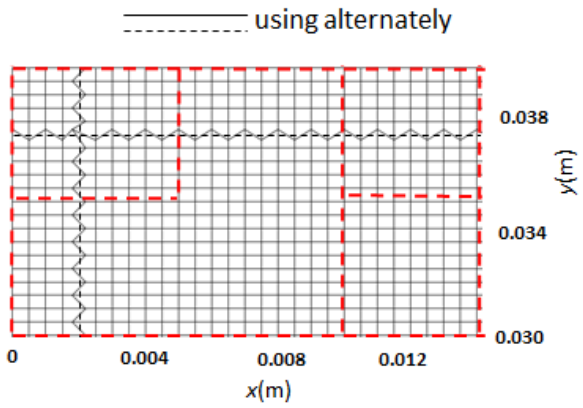

(c)

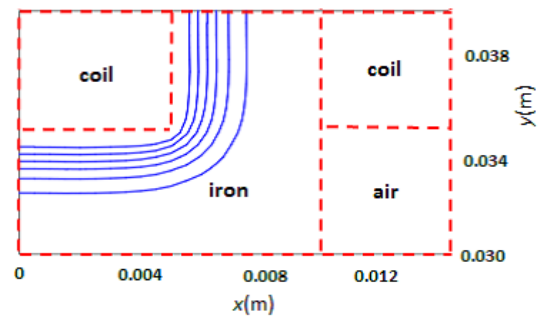

(d)

Fig. 5. Magnetic flux lines obtained by: (a) the conventional FEM, and (b) the space-time FEM using the triangular prism element. (c) Shape of elements (d) obtained by the space-time FEM using elements as in (c) 
solid and dashed lines alternate along the temporary direction and are connected by the space-time hexahedral elements. This space-time grid yields flux lines depicted in Fig. 5(d). The vector potential distributions corresponding to Figs. 5(a) (b) and (d) along the $x$-direction at $y=0.038 \mathrm{~m}$ are compared in Fig. 6. The flux lines and vector potential given by the space-time FEM agree with those simulated by the conventional FEM.

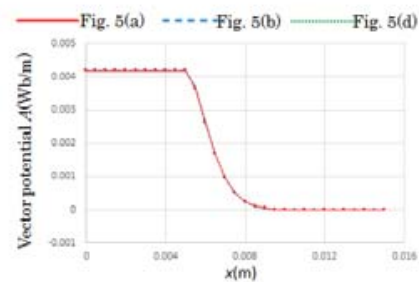

Fig. 6. Distributions of vector potential along $y=0.038 \mathrm{~m}$ corresponding to Fig. 5

\section{Moving conductor}

The field of a moving conductor (Fig. 7) was analyzed using the 3D space-time FEM, where the conductivity and relative permeability of the moving conductor are $59 \times 10^{6} \mathrm{~S} / \mathrm{m}$ and 1 ; the velocity of the moving conductor is $100 \mathrm{~m} / \mathrm{s}$. The natural boundary condition is imposed at the symmetric

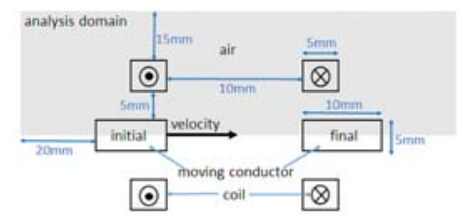

Fig. 7. Field with a moving conductor

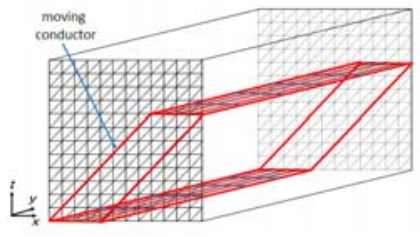

Fig. 8. Representation of a moving conductor by triangular prism elements boundaries, and $A=0$ at the outer boundaries. Fig. 8 shows how to represent the moving conductor using the triangular prism element.

Fig. 9 depicts magnetic flux lines when the moving conductor reaches the center of the coils. The lines were obtained using conventional FEM with fixed and moving coordinates and using the space-time FEM with triangular prism elements. The vector potential distributions corresponding to Figs. 9(a)-(c) along the $x$ - direction at $y=$ $0.003(\mathrm{~m})$ are compared in Fig. 10. The space-time FEM yields very similar flux lines and vector potential to those obtained using the conventional FEM with a moving coordinate system. The flux lines concentrate in front of the conductor giving a deceleration force. The conventional FEM with a fixed coordinate system yields slightly different flux lines from other methods. This is because the moving coordinate system gives a more accurate result than the fixed

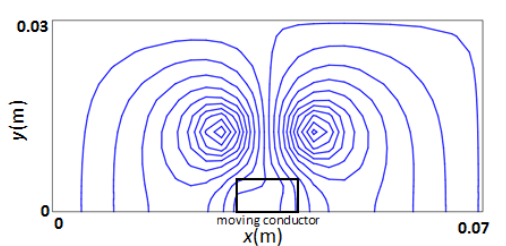

(a)

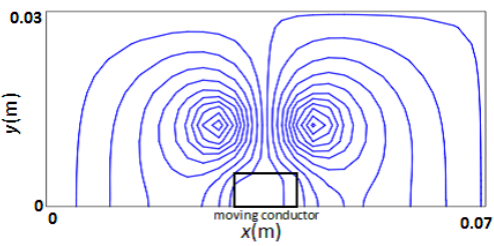

(b)

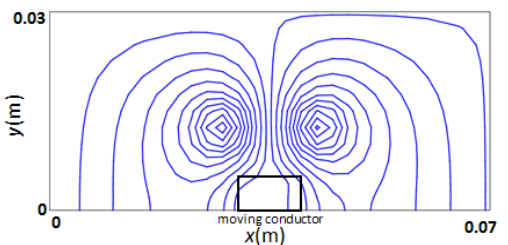

(c)

Fig. 9. Magnetic flux lines obtained using: (a) conventional FEM with stationary coordinate system, (b) conventional FEM with a moving coordinate system, and (c) the space-time FEM

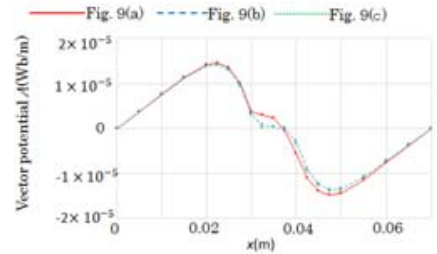

Fig. 10. Distributions of vector potential along $y=0.003 \mathrm{~m}$ corresponding to Fig. 9.

system [2]. In contrast, the space-time finite element is independent of the coordinate system.

\section{CONCLUSION}

The relationship between the Galilean transformation and the space-time finite element was considered in regard to treating a moving conductor with an electromotive force and a temporal-variant spatial grid. The space-time FEM using triangular prismatic elements reproduces accurately the eddy current field with and without a moving conductor.

\section{REFERENCES}

[1] K. Muramatsu, T. Nakata, N. Takahashi and K. Fujiwara, "Comparison of coordinate systems for eddy current analysis in moving conductors," IEEE Trans. Magn., vol. 28, pp. 1186-1189, Mar. 1992.

[2] K. Yamazaki, "Generalization of 3D eddy current analysis for moving conductors due to coordinate systems and gauge conditions," IEEE Trans. Magn., vol. 33, pp. 1259-1262, Mar. 1997.

[3] A. J. Butler and Z. J. Cendes, "Space-time finite elements derived by convolution for the efficient solution of transient eddy current problems," IEEE Trans. Magn., vol. 24, pp. 2688-2690, Nov. 1988.

[4] T. Renyuan, L. Feng, L. Yan and C. Xiang, "Analysis of transient nonlinear eddy current fields by space-time finite element method," IEEE Trans. Magn., vol. 34, pp. 2577-2580, Sept. 1998.

[5] S. Gyimóthy, A. Vágvölgyi, and I. Sebestyén, “Application of optimally distorted finite elements for field calculation problems of electromagnetism," IEEE Trans. Magn., vol. 38, pp. 365-368, Mar. 2002 . 